hep-ph/9906253 (revised version)

Phys. Lett. B 461 (1999) 248, ibid 471 (2000) 471E

\title{
Heavy Majorana neutrino production at electron-muon colliders
}

\author{
G. Cvetič \\ Dept. of Physics, Universität Bielefeld, 33501 Bielefeld, Germany; \\ Dept. of Physics, Universität Dortmund, 44221 Dortmund, Germany \\ C.S. Kiml \\ Dept. of Physics, Yonsei University, Seoul 120-749, Korea
}

\begin{abstract}
Possibilities for detecting heavy Majorana neutrinos ( $N$ 's) at future $e \mu$ colliders are investigated. In contrast to the $e^{-} e^{+}$colliders (LEP200 and NLC), the center-of-mass (CMS) energies achieved at $e \mu$ colliders can be much higher and the $Z$-mediated $s$ channel is excluded automatically. This opens the attractive possibility of having high production cross sections for $N$ 's and at the same time probing only the strength of charged current couplings of $N$ 's $(N W e$ and $N W \mu$ ). The production cross sections and the expected numbers of events for the reaction $e^{\mp} \mu^{ \pm} \rightarrow N N \rightarrow W^{ \pm} \ell^{\mp} W^{ \pm} \ell^{\prime \mp}$ are calculated for various masses $M$ of the Majorana neutrinos and for the CMS energies $\sqrt{s}=0.5-6.0 \mathrm{TeV}$. The values of the charged current coupling parameters are set equal to their present upper bounds. We obtain reasonably high production cross sections. Further, the effects of the off-shell intermediate $N$ 's turn out to be significant only at $\sqrt{s} \approx 2 \mathrm{TeV}$.
\end{abstract}

PACS number(s): 14.60.St, 11.30.Fs, 13.10.+q, 13.35.Hb

*e-mail: cvetic@physik.uni-bielefeld.de

†e-mail: kim@cskim.yonsei.ac.kr; http://phya.yonsei.ac.kr/ cskim/ 


\section{INTRODUCTION}

One of the basic questions in high energy physics is: Are neutrinos Dirac or Majorana particles? In the absence of right-handed currents, it is virtually impossible to discern the nature of the light neutrinos [1]. However, if heavy neutrinos $\left(M \gtrsim 10^{2} \mathrm{GeV}\right)$ exist, then present and, even more so, future experiments could establish whether such neutrinos are Majorana or Dirac. Many theoretical investigations have been carried out [2] into the production cross sections of heavy Majorana neutrinos ( $N$ 's) as predicted by various models, via $e^{-} e^{+}, e p$ and $p p$ collisions, and mostly within specific models or classes of models. As a rule, it has been assumed that the center-of-mass (CMS) energy $\sqrt{s}$ in the process is high enough for the production of on-shell (OS) heavy $N$ 's $\left(e^{+} e^{-}, e p, p p \rightarrow N N\right)$. Further, the effects of the off-shell (nOS) $N$ 's have been ignored. We note that the detection of $N N$ pair production events can be realized by identifying the final decay products (on-shell) of the Majorana neutrinos - primarily the leptons $\ell_{j}$ 's and the $W$ 's. Thus, the reactions $\ell^{-} \ell^{\prime+} \rightarrow N N \rightarrow W^{ \pm} \ell_{i}^{\mp} W^{ \pm} \ell_{j}^{\mp} \rightarrow$ jets $+\ell_{i}^{\mp} \ell_{j}^{\mp}$, which violate the total lepton number, would be a clear signal of the Majorana character of the intermediate neutrinos (the latter need not be on-shell). Here, $\ell$ and $\ell^{\prime}$ are the two initial leptons, either $e^{-} e^{+}$or $e \mu$.

In a previous paper [3], we demonstrated the importance of taking into account the contributions of off-shell intermediate $N$ 's in such processes, in $e^{-} e^{+}$colliders such as LEP200 and future Linear Colliders. Several advantages of $e \mu$ colliders were pointed out by the authors of [4]. In the present paper, we present some results of calculations for the aforementioned reactions for $e \mu$ colliders. The main advantages of the $e \mu$ colliders, in contrast to the $e^{-} e^{+}$colliders, from our perspective, are the following:

- $\mu$ 's can easily be accelerated to very high energies due to reduced synchrotron radiation loss (since $m_{\mu} \gg m_{e}$ );

- the $s$ channel ( $Z$-mediated) is not present now.

Due to the first point, the event rates can be increased because of the increased center-ofmass (CMS) energy $\sqrt{s}$ of the process. Due to the second point, the number of parameters on which the production rate depends is reduced since only the couplings of $N$ 's to charged currents contribute ( $\mu W N$ and $e W N$ couplings). The second point does not apply in the case of $\mu^{-} \mu^{+}$colliders, the case which we therefore do not consider in the present paper.

As in the previous paper [3], our analysis is rather general, in the sense that we do not restrict ourselves to any specific (classes of) models. Further, we use basically the same set of programs developed by us there, this time adjusted to the case of $e \mu$ colliders. We stress that, in contrast to the available literature, our numerical calculations include the effects of off-shell (nOS) intermediate $N$ 's on the cross sections. This enables us to investigate deviations from the previously known cross sections (with two on-shell intermediate $N$ 's), in the "2OS" kinematic region $\left(\sqrt{s}>2 M>2 M_{W}\right)$ where both intermediate $N$ 's can, but need not, be on shell (OS) - these deviations are often referred to as "finite width effects". Further, our general expressions allow us to calculate the cross sections also in the "1OS"

1 This was the case also in our previous paper [3]. 
kinematic region $\left(2 M>\sqrt{s}>M+M_{W}\right)$ where at most one intermediate $N$ can (but need not) be on shell, and even in the "nOS" region $\left(M+M_{W}>\sqrt{s}\right)$ where both intermediate $N$ 's always have to be off shell (nOS). For various choices of physical and kinematic parameters, we calculate the total cross section for the mentioned lepton-number violating reactions and the corresponding expected numbers of events for $e \mu$ colliders.

\section{REACTION AMPLITUDE AND ITS SQUARE}

Our starting point is the rather general Lagrangian density for the couplings of the heavy Majorana neutrinos $N$ with $W^{\prime}$ 's and light leptons $\ell_{j}\left(j=1,2,3 ; \ell_{1}^{-}=e^{-}, \ell_{2}^{-}=\mu^{-}, \ell_{3}=\tau^{-}\right)$:

$$
\mathcal{L}_{N \ell W}(x)=-\frac{g}{2 \sqrt{2}} \sum_{j=1}^{3} B_{L}^{(j)} \bar{\ell}_{j}(x) \gamma^{\mu}\left(1-\gamma_{5}\right) N(x) W_{\mu}^{(-)}+\text {h.c. }
$$

where $B_{L}^{(j)}$ 's can be regarded, at first, as basically free parameters (later we discuss some possible constraints on $B_{L}^{(j)}$ 's). In Eq. (11), $g$ is the standard $S U(2)_{L}$ gauge coupling parameter. We mention that the $N \ell W$ couplings could include, in principle, the right-handed parts, but this possibility will not be considered here. Our choice would thus appear to suggest that the considered $N$ (mass eigenstate) is primarily sequential, i.e., with the standard $S U(2)_{L} \times U(1)_{Y}$ assignments. However, our choice can approximately describe also many other scenarios, including the one in which $N$ has an admixture of $S U(2)_{L}$-singlet component, provided the right-handed coupling analogous to (1) is reasonably suppressed. Further, it turns out that parameters $B_{L}^{(j)}$ 's affect the final results only via the combinations

$$
H^{\prime} \equiv\left|B_{L}^{(1) *} B_{L}^{(2)}\right|, \quad H \equiv \sum_{j=1}^{3}\left|B_{L}^{(j)}\right|^{2}
$$

We will restrict ourselves to considering the reactions $e^{-} \mu^{+} \rightarrow N N \rightarrow W^{ \pm} \ell_{i}^{\mp} W^{ \pm} \ell_{j}^{\mp}(\rightarrow$ jets $\left.+\ell_{i}^{\mp} \ell_{j}^{\mp}\right)$, which represent an explicit violation of the total lepton number - characteristic for the intermediate heavy Majorana neutrinos. The reaction involves only the $t+u$ (shortly: $t u$ ) channel - cf. Fig. 1. The $s$ channel $(Z$-mediated) is not allowed.

For the calculation of the invariant amplitude $\mathcal{M}_{f i}$ (shortly: $\mathcal{M}$ ) for the $t u$ channel, [] we used the 4-component spinors $u^{(\alpha)}(q) \equiv u(q \alpha)$ and $v^{(\alpha)}(q) \equiv v(q \alpha)$ as defined by Itzykson and Zuber [5] (I.Z.), but with the normalization convention as given in [6], i.e., $u=\sqrt{2 m_{f}} u_{\text {I.Z. }}$. and $v=\sqrt{2 m_{f}} v_{\text {I.Z. }}$, where $m_{f}$ is the mass of the fermion. Further, for the spin component quantum numbers of spinors, we use the following notational convention for tildes: $\widetilde{\alpha}=$ $1,2 \Leftrightarrow \alpha=2,1$. The $t u$-channel amplitude $\mathcal{M}^{(t u)}$ is

$$
\begin{aligned}
& \mathrm{i} \mathcal{M}^{(t u)}=\frac{4 M A^{(t u)}}{\left[\left(p-p_{\ell}-p_{w}\right)^{2}-M_{W}^{2}+\mathrm{i} \Gamma_{W} M_{W}\right]}(-1)^{\bar{\alpha}_{\ell}} P_{N}\left(p_{\ell} p_{w}\right) P_{N}\left(\bar{p}_{\ell} \bar{p}_{w}\right) \times \\
& \left\{\left[\bar{u}\left(p_{\ell} ; \alpha_{\ell}\right)\left(\notin p_{w}+2 p_{\ell} \cdot \varepsilon\right) \gamma^{\nu}\left(1-\gamma_{5}\right) u(p ; \alpha)\right]\left[\bar{v}(\bar{p} ; \bar{\alpha}) \gamma_{\nu}\left(1+\gamma_{5}\right) \notin\left(1+\gamma_{5}\right) v\left(\bar{p}_{\ell} ; \widetilde{\bar{\alpha}}_{\ell}\right)\right]\right. \\
& \left.+\frac{\left(p_{\ell}+p_{w}\right)^{2}}{M_{W}^{2}}\left[\bar{u}\left(p_{\ell} ; \alpha_{\ell}\right) \notin\left(1-\gamma_{5}\right) u(p ; \alpha)\right]\left[\bar{v}(\bar{p} ; \bar{\alpha})\left(\bar{p}_{w} \bar{\ddagger}+2 \bar{p}_{\ell} \cdot \bar{\varepsilon}\right)\left(1+\gamma_{5}\right) v\left(\bar{p}_{\ell} ; \widetilde{\bar{\alpha}}_{\ell}\right)\right]\right\}+\ldots
\end{aligned}
$$

\footnotetext{
${ }^{2} \mathcal{M}_{f i}$ is defined by [6]: $\langle$ fin $|S| i n\rangle \equiv \mathrm{i}(2 \pi)^{4} \delta^{(4)}\left(P_{\text {in }}-P_{\text {fin }}\right) \mathcal{M}_{f i}$, for $\mid$ fin $\rangle \neq|i n\rangle$.
} 
Here we use the notations of Fig. 11. Further, $\not d \equiv q_{\nu} \gamma^{\nu} ; \varepsilon_{\nu} \equiv \varepsilon_{\nu}^{(\lambda)}\left(p_{w}\right)$ and $\bar{\varepsilon}_{\nu} \equiv \varepsilon_{\nu}^{(\bar{\lambda})}\left(\bar{p}_{w}\right)$ are the real polarization vectors [5] of the final $W$ 's, with polarizations $\lambda, \bar{\lambda}=1,2,3$. $M$ is the mass of $N$ 's. Parameter $A^{(t u)}$ in Eq. (3) contains the strengths of the couplings of the two Majorana neutrinos in the $t u$ channel

$$
A^{(t u)}=\frac{g^{4}}{64} B_{L}^{(1) *} B_{L}^{(2)} B_{L}^{(i)} B_{L}^{(j)} \mathrm{i} \lambda_{M},
$$

where $\lambda_{M}$ is the phase factor in the Fourier decomposition of the Majorana field $N(x)$ (cf. [7]; $\left|\lambda_{M}\right|^{2}=1$ ). $P_{N}$ in Eq. (3) is the (scalar) denominator of the propagator of $N$

$$
P_{N}\left(p_{\ell} p_{w}\right)=1 /\left[\left(p_{\ell}+p_{w}\right)^{2}-M^{2}+\mathrm{i} M \Gamma_{N}\right],
$$

where $\Gamma_{N}$ is the total decay width of $N$. The dots in Eq. (3) at the end stand for three analogous terms, obtained from the expression explicitly written above by replacements: (I) $\left(p_{w}, \varepsilon\right) \leftrightarrow\left(\bar{p}_{w}, \bar{\varepsilon}\right)$; (II) $\left(p_{\ell}, \alpha\right) \leftrightarrow\left(\bar{p}_{\ell}, \bar{\alpha}\right)$ and overall factor $(-1)$; (III) combined replacements (I) and (II). In expression (3) we neglected the masses of all the charged leptons appearing $(e, \mu, \tau)$.

We can reexpress any of the above terms in $\mathcal{M}^{(t u)}$ in alternative forms, by applying the following general identities:

$$
-\mathrm{i} \gamma^{2} u(q \alpha)^{*}=(-1)^{\alpha} v(q \widetilde{\alpha}),-\mathrm{i} \gamma^{2} v(q \alpha)^{*}=(-1)^{\widetilde{\alpha}} u(q \widetilde{\alpha}),
$$

and $(\not \not b)^{T}=-\gamma^{0} \gamma^{2}(\not b \not) \gamma^{2} \gamma^{0}$, where the Dirac basis and the conventions of [5] are used for $\gamma^{\mu}$ 's. We use (6), for example, if we want to employ, in scalar expressions in square brackets of Eq. (3) $), u(\bar{p} ; \overline{\bar{\alpha}})$ and $\bar{u}\left(\bar{p}_{\ell} ; \bar{\alpha}_{\ell}\right)$ instead of $\bar{v}(\bar{p} ; \bar{\alpha})$ and $v\left(\bar{p}_{\ell} ; \tilde{\bar{\alpha}}\right)$. Such transformations are very convenient when calculating $\left\langle|\mathcal{M}|^{2}\right\rangle=\left\langle\left|\mathcal{M}^{(t u)}\right|^{2}\right\rangle$. Using the afore-mentioned tranformations judiciously, we can always end up with traces of $4 \times 4$ matrices involving $u(q, \beta) \bar{u}(q, \beta)=\not$ and/or $v(q, \beta) \bar{v}(q, \beta)=\not q(q=p, \bar{p}, \ldots ; \beta=\alpha, \bar{\alpha}, \ldots=1,2)$. We performed these traces by writing programs in Mathematica [8] and employing there subroutine Tracer [9]. Single traces involve up to fourteen $\gamma^{\mu}$ matrices and $\left(1-\gamma_{5}\right)$ matrix.

The expressions for $\left\langle|\mathcal{M}|^{2}\right\rangle$, obtained in the way described above, were then rewritten with short notations for various scalar products and for the contracted Levi-Civita tensors. They were then translated into an optimized FORTRAN form by employing Maple [10], and fed into our main program for calculation of the total cross sections $\sigma$. The integrand $\left\langle|\mathcal{M}|^{2}\right\rangle$ is a long expression, extending over tens of pages when printed out. The main program uses subroutines rambo [1] and vegas [12] for numerical integration.

\section{NUMERICAL CALCULATIONS AND RESULTS}

We stress that our general program can now deal with all kinematic situations: 1.) those where both intermediate $N$ 's can (but need not) be on shell [2OS kinematic region: $\sqrt{s}>2 M>2 M_{W}$;

2.) those where only at most one $N$ can (but need not) be on shell [1OS kinematic region: $\left.2 M>\sqrt{s}>M+M_{W}\right]$

3.) those where both $N$ 's must be off shell [nOS kinematic region: $M+M_{W}>\sqrt{s}$. 
However, by the same methods as described above, we also calculated the amplitudes $\mathcal{M}$ and their averaged squares $\left\langle|\mathcal{M}|^{2}\right\rangle$ when one $N$, or both $N$ 's, are explicitly put on shell (1OS, 2OS expressions, respectively). These expressions turn out to be much shorter than the general (nOS) expressions with both $N$ 's propagating. Analogously as for the nOS expressions (cf. previous Section), also the 1OS and 2OS expressions for $\left\langle|\mathcal{M}|^{2}\right\rangle$ were calculated with help of the subroutine Tracer and were then fed into separate FORTRAN programs where they were combined with the rambo and vegas subroutines. These (1OS, 2OS) programs allow us to calculate the sum of cross sections for the considered reactions $e^{-} \mu^{+} \rightarrow N N \rightarrow W^{+} \ell_{i}^{-} W^{+} \ell_{j}^{-}(i, j=1,2,3)$ where now one, or both, intermediate $N^{\prime}$ s are explicitly on shell. In fact, the $1 \mathrm{OS}$ expression for $\left\langle|\mathcal{M}|^{2}\right\rangle$, for the sum of reactions $e^{-} \mu^{+} \rightarrow N N(O S) \rightarrow W^{+} \ell_{i}^{-} N(O S)(i=1,2,3)$, is multiplied simply by the branching ratio $\mathrm{Br}$ for the sum of the decay modes $N(O S) \rightarrow W^{+} \ell_{j}^{-}(j=1,2,3)$; the 2OS expression for the reaction $e^{-} \mu^{+} \rightarrow N(O S) N(O S)$ is multiplied by $(B r)^{2}$.

For numerical calculations, the input were values of $\sqrt{s}, M$, and of the "mixing" parameters $H^{\prime}$ and $H$ [cf. Eqs. (1)-(2)]. Parameter $H^{\prime}$ is a combined measure of the strengths of the $e W N$ and $\mu W N$ couplings and it affects the $(t u)$ amplitude crucially $\left(\propto H^{\prime}\right)$. Parameter $H$ affects the total $\left\langle|\mathcal{M}|^{2}\right\rangle$ which is then formally proportional to $H^{2}$ (once $H^{\prime}$ is fixed). This is so because we sum over all the flavors of the two final light leptons $\ell_{i}^{-}, \ell_{j}^{-}$. All in all, in $\left\langle|\mathcal{M}|^{2}\right\rangle$ we thus average over the spin components $(\alpha, \bar{\alpha})$ of the initial $e^{-}$and $\mu^{+}$(factor $1 / 4)$, sum over the polarizations $(\lambda, \bar{\lambda})$ of the two final $W^{+}$'s and over the spin components $\left(\alpha_{\ell}, \bar{\alpha}_{\ell}\right)$ and the flavors $(i, j=1,2,3)$ of the two final light leptons. In the general (nOS) expression, we have to include an additional factor $1 / 2$ to avoid double-counting of the two $W^{+}$'s, and factor $1 / 2$ to avoid double-counting of the final leptons (i.e., of $\ell_{i}^{-} \ell_{i}^{-}$, or of twice $\ell_{i}^{-} \ell_{j}^{-}$when $\left.i \neq j\right)$. The $\left\langle|\mathcal{M}|^{2}\right\rangle$ thus defined can then be integrated over the entire phase space of the final particles (massive $W^{\prime}$ 's and massless $\ell$ ), and there is no double-counting in the obtained total $\sigma$.

Further, the total decay width $\Gamma_{N}$ of $N$ 's, appearing in the denominators of their propagators, was determined by us at the tree level and under the assumption that the only (dominant) decay modes are $N \rightarrow W^{ \pm} \ell_{j}^{\mp}(j=1,2,3)$. Incidentally, this means that the branching ratio for the sum of the decay modes $N \rightarrow W^{+} \ell_{j}^{-}(j=1,2,3)$ is simply $\mathrm{Br}=1 / 2$, the value that is then also used in the 1OS and 2OS programs for cross sections (cf. discussion above). In this framework, we have $\Gamma_{N} \propto H$, i.e., $H$ is the decay width parameter of the $N$ 's.

In large classes of models, in which heavy neutrinos are sequential or have exotic $S U(2) \times$ $U(1)$ assignments, the values of the "mixing" parameters $H^{\prime}$ and $H$ [cf. Eq. (2)] are severely restricted by available experimental data (LEP and low-energy data) [13,14]:

${ }^{3}$ In the simpler $1 \mathrm{OS}$ and $2 \mathrm{OS}$ expressions for $\left\langle|\mathcal{M}|^{2}\right\rangle$, these factors are different. For the 2OS expression, the factor is $(1 / 2)$ because the two intermediate on-shell $N$ 's are indistinguishable, and thus their decay product pairs $\left(W^{+} \ell_{i}^{-}, W^{+} \ell_{j}^{-}\right)$are also kinematically indistinguishable. For the $1 \mathrm{OS}$ expression, in the $1 \mathrm{OS}$ kinematic region, all four final particles are distinguishable via kinematics (i.e., invariant masses) of $W^{+} \ell^{-}$pairs, and the factor is 1 ; in the $2 \mathrm{OS}$ kinematic region, the two decay product pairs $\left(W^{+} \ell_{i}^{-}, W^{+} \ell_{j}\right)$ become kinematically indistinguishable, and the factor is $(1 / 2)$. 


$$
H^{\prime} \lesssim 0.0155, \quad H \lesssim 0.122
$$

For orientation, we used for the values of $H^{\prime}$ and $H$ these two upper bounds. The numerical results for the cross sections $\sigma$ of the reactions $e^{-} \mu^{+} \rightarrow N N \rightarrow W^{+} \ell_{i}^{-} W^{+} \ell_{j}^{-}$(summed over $i, j=1,2,3)$ are depicted in Figs. 2-6.

In Figs. 205, the cross sections $\sigma$ are presented as functions of the Majorana neutrino mass $M$, at five different CMS reaction energies $\sqrt{s}=0.5,1,2,6 \mathrm{TeV}$, respectively. Calculations were performed in various kinematic regions (nOS, 1OS, 2OS regions) with the general (nOS) program which has the propagating intermediate $N$ 's with finite width [cf. Eqs. (3), (5)]. Further, in the $1 \mathrm{OS}$ and $2 \mathrm{OS}$ kinematic regions, the cross sections were calculated also with the 1 OS program (in which one $N$ is explicitly on shell). In the 2 OS kinematic region, the cross sections were performed in addition with the $2 \mathrm{OS}$ program (in which both $N$ 's are explicitly on shell). Comparing the results of the general nOS program with those of the 1OS and $2 \mathrm{OS}$ programs, we see from these Figures that the contributions of the configurations with off-shell Majorana neutrinos become important only at high $\sqrt{s} \geq 2 \mathrm{TeV}$. For example, when $\sqrt{s}=2.0 \mathrm{TeV}$, the results of the nOS program in the $2 \mathrm{OS}$ kinematic region $(M<\sqrt{s} / 2)$ differ from those of the 2OS (and 1OS) programs already by up to $30 \%$. One of the reasons for this trend lies in the fact that higher $\sqrt{s}$ (at a given $M$ ) mean that more off-shell configurations are available for the intermediate $N$ 's. Further, in Figs. 24 we see the slope increase associated with the change of kinematic regions: at $M \approx \sqrt{s} / 2$ (onset of the $2 \mathrm{OS}$ region). In Fig. 5, only the $2 \mathrm{OS}$ kinematic region is included.

In connection with Figs. 2-5, one technical difficulty with the general (nOS) program should be mentioned. The results of the nOS program were not depicted in the lowest parts of the 2OS kinematic regions ( $M$ much lower than $\sqrt{s} / 2)$. In these regions of low $M$, the nOS program requires exceedingly high statistics to avoid large numerical uncertainties. These numerical uncertainties arise because, for low $M$ 's $\left(\sim M_{W}\right)$ only a very limited part of the rambo-generated phase space of final particles contributes to the cross section (that would be true even when $\Gamma_{N}$ were, artificially by hand, prevented from approaching the zero value when $\left.M \rightarrow M_{W}\right)$. An additional important reason for these uncertainties lies in the fact that the integrand becomes very singularly peaked in small subregions of that mentioned part of the phase space. This is so because $\Gamma_{N}$ becomes small for small $M$ 's, and consequently the absolute square of expression (5) is then almost equal to $\left[\pi /\left(M \Gamma_{N}\right)\right] \delta\left[\left(p_{\ell}+p_{w}\right)^{2}-M^{2}\right]$.

In Fig. 6] we plot the cross sections as function of the CSM energy $\sqrt{s}$, at fixed chosen values of the Majorana mass $M=200,400$ and $600 \mathrm{GeV}$. Here we see that, at a given Majorana neutrino mass $M$, the full cross section usually (but not always) increases with increasing $\sqrt{s}$. At high CSM energies $\sqrt{s}>2 M$ (2OS kinematic region), the cross section changes only slowly with increasing $\sqrt{s}$. In Fig. 6, for $M=200 \mathrm{GeV}$ only results of the $2 \mathrm{OS}$ program (in which both N's are put on shell) are displayed, since the general (nOS) and the 1OS programs show up the afore-mentioned numerical instabilities and large numerical uncertainties at so low values of $M$. Even for $M=400 \mathrm{GeV}$, the nOS program has significant numerical uncertainties $(\sim 10 \%)$ for $\sigma$ in the region $\sqrt{s}=1-2 \mathrm{TeV}$, but these results were included nonetheless.

One may ask how the depicted results change once we change the values of the "mixing" parameters $H^{\prime}$ and $H$ [cf. Eqs. (2), (7)]. It turns out that, for the $2 \mathrm{OS}$ kinematic region $\left(\sqrt{s}>2 M>2 M_{W}\right)$, the cross sections (and distributions) as given by the general (nOS) program depend on the parameter $H$ only weakly. This can be understood first in the 
formal way, because the absolute square of each of the two intermediate $N$-propagators (when $\Gamma_{N} \ll M$ ) is approximately proportional to $\Gamma_{N}^{-1} \propto H^{-1}$ (cf. previous discussion), while the sum of the squares of the amplitudes without $N$-propagators is proportional to $H^{2}-$ the latter because of the two decay vertices $N \rightarrow W^{+} \ell_{j}^{-}$and $N \rightarrow W^{+} \ell_{i}^{-}$(cf. Fig. 1) which are squared and summed $(i, j=1,2,3)$. This approximate $H$-independence can be expected on physical grounds: These cross sections are, for not too high $\sqrt{s}$, dominated by the configurations with two on-shell $N$ 's; but the absolute squares of amplitudes for $e^{-} \mu^{+} \rightarrow N(O S) N(O S)$ [combined with the decay branching ratios $(1 / 2)^{2}$ for the $N(O S)^{\prime}$ s] are exactly $H$-independent. Parameter $H$, being responsible for the finite width (note: $\left.\Gamma_{N} \propto H\right)$, is apparently one of the two principal parameters responsible in the 2OS kinematic region for the deviation of the cross section from the pure 2OS cross section (the other is $\sqrt{s})$. On the other hand, in the 1 OS kinematic region $\left(2 M>\sqrt{s}>M+M_{W}\right)$, the $H$ dependence of the cross section becomes quite strong (approximately $\propto H$ ), and in the nOS region $\left(M+M_{W}>\sqrt{s}\right)$ even more so $\left(\propto H^{2}\right)$.

On the other hand, the dependence of the cross sections on the parameter $H^{\prime}$ [cf. Eqs. (2), (田)] is always strong $\left(\propto H^{\prime 2}\right)$.

\section{SUMMARY AND CONCLUSIONS}

We investigated the reactions $e^{-} \mu^{+} \rightarrow N N \rightarrow W^{+} \ell^{-} W^{+} \ell^{\prime-}$ where the intermediate $N$ 's are heavy Majorana neutrinos (with mass $M \gtrsim 10^{2} \mathrm{GeV}$ ) and $\ell, \ell^{\prime}$ are light charged leptons $(e, \mu, \tau)$. These processes violate the total lepton number and, if detected, would represent a clear signal of the Majorana character of (heavy) neutrinos. In contrast to the expressions available in the literature so far where the intermediate $N$ 's are taken to be on shell, we calculated the amplitudes of these processes by employing finite width propagators of the $N$ 's. This enabled us to calculate numerically the full cross section for the reactions, i.e., including the effects of the off-shell intermediate $N^{\prime}$ 's. In contrast to the analogous reactions in $e^{-} e^{+}$(and $\left.\mu^{-} \mu^{+}\right)$colliders, the considered reactions do not include a ( $Z$-mediated) $s$ channel, but only a ( $W$-mediated) tu channel, thus allowing us to reduce the number of relevant unknown coupling parameters. If the CMS process energies $\sqrt{s}$ are large $(\geq 2$ $\mathrm{TeV}$ ), the effects of the off-shell intermediate $N$ 's can be significant even in the 2OS kinematic region, i.e., in the region $\sqrt{s}>2 M\left(>2 M_{W}\right)$ where the configurations with both $N$ 's being on shell are allowed. Further, the number of such events at future $e \mu$ colliders would be in general high enough for detection. For example, if assuming the integrated luminosity $10^{4} \mathrm{pb}^{-1}$, and Majorana mass $M=200 \mathrm{GeV}(400 \mathrm{GeV})$, we would get about 10, 24, 29, 31 $(0,27,32,188)$ events at $\sqrt{s}=0.5,1.0,2.0$ and $6.0 \mathrm{TeV}$, respectively. These numbers have to be multiplied by two if we include also the events with two positively charged leptons in the final state (the latter events also violate the total lepton number conservation, of course). We should stress that we used the highly suppressed values of the "mixing" parameters $H^{\prime}=0.0155$ and $H=0.122$. These values are the maximal values allowed in theories where the heavy neutrinos are either sequential or have exotic $S U(2) \times U(1)$ assignments. If these bounds do not apply, the number of events can increase dramatically since it is proportional to $H^{\prime 2}$.

In the paper, we ignored the questions connected with the experimental difficulties of detecting the discussed process unambiguously. In particular, there are problems connected 
with the identification of the (on-shell) $W^{\prime}$ 's and some of the light charged leptons $(\tau$ 's). Further, we ignored the possibility that the Majorana neutrino mass $M$ is very low - below $M_{W}$ (the $W^{+}$'s are then off shell) [15]. In such interesting cases of low $M$, however, additional experimental problems would arise in the identification of the process since the two $W^{+}$'s are now intermediate off-shell particles.

\section{Acknowledgments:}

We would like to thank V. Barger for his helpful comments. G.C. acknowledges support by the BMBF project 05-7DO93P7 during the initial stage of this work, and he thanks

Prof. D. Schildknecht for the hospitality of Bielefeld University during the completion of this work. The work of C.S.K. was supported in part by KRF Non-Directed-ResearchFund, Project No. 1997-001-D00111, in part by the BSRI Program, Ministry of Education, Project No. 98-015-D00061, in part by the KOSEF-DFG large collaboration project, Project No. 96-0702-01-01-2. 


\section{REFERENCES}

[1] B. Kayser, Phys. Rev. D 26, 1662 (1982).

[2] F. del Aguila, E. Laerman, and P. Zerwas, Nucl. Phys. B 297 (1988) 1; E. Ma and J. Pantaleone, Phys. Rev. D 40 (1989) 2172; W. Buchmüller and C. Greub, Nucl. Phys. B 363 (1991) 345; ibid 381 (1992) 109; J. Maalampi, K. Mursula, and R. Vuopionperä, Nucl. Phys. B 372 (1992) 23; A. Datta and A. Pilaftsis, Phys. Lett. B 278 (1992) 162; A. Datta, M. Guchait, and A. Pilaftsis, Phys. Rev. D 50 (1994) 3195; J. Gluza and M. Zrałek, Phys. Rev. D 48 (1993) 5093; ibid 51 (1995) 4695; ibid 51 (1995) 4707; A. Denner, H. Eck, O. Hahn, and J. Küblbeck, Nucl. Phys. B 387 (1992) 467; J. Kogo and S.Y. Tsai, Prog. Theor. Phys. 86 (1991) 183; A. Hoefer and L.M. Sehgal, Phys. Rev. D 54 (1996) 1944.

[3] G. Cvetič, C.S. Kim, and C.W. Kim, Phys. Rev. Lett. 82 (1999) 4761.

[4] S.Y. Choi, C.S. Kim, Y.J. Kwon, and S.-H. Lee, Phys. Rev. D 57 (1998) 7023; V. Barger, S. Pakvasa, and X. Tata, Phys. Lett. B 415 (1997) 200.

[5] C. Itzykson and J.-B. Zuber, Quantum field theory, McGraw-Hill Inc., 1980.

[6] M.E. Peskin and D.V. Schroeder, An introduction to quantum field theory, AddisonWesley, 1995; V.D. Barger and R.J.N. Phillips, Collider Physics, Addison-Wesley, 1987.

[7] B. Kayser, F. Gibrat-Debu, and F. Perrier, The physics of massive neutrinos, World Scientific, 1989.

[8] S. Wolfram, Mathematica, Addison-Wesley, 1991.

[9] M. Jamin and M.E. Lautenbacher, Comp. Phys. Comm. 74 (1993) 265.

[10] The Maple Handbook: Maple V release 4, ed. by D. Redfern, Springer-Verlag, 1996.

[11] R. Kleiss, W.J. Stirling, and S.D. Ellis, Comp. Phys. Comm. 40 (1986) 359.

[12] G.P. Lepage, J. Comp. Phys. 27 (1978) 192.

[13] P. Langacker and D. London, Phys. Rev. D 38 (1988) 886.

[14] G. Bhattacharyya and A. Datta, Mod. Phys. Lett. A 6 (1991) 2921; E. Nardi, E. Roulet, and D. Tommasini, Nucl. Phys. B 386 (1992) 239.

[15] A. Datta, private communication. 


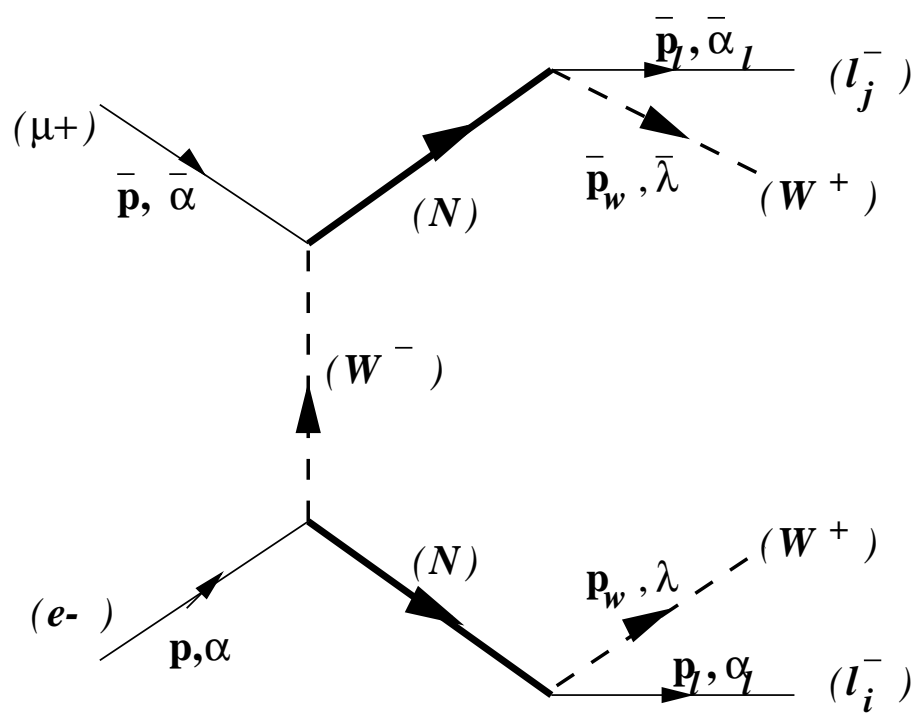

FIG. 1. Reaction $e^{-}(p, \alpha) \mu^{+}(\bar{p}, \bar{\alpha}) \rightarrow N^{*} N^{*} \rightarrow W^{+}\left(p_{w}, \lambda\right) W^{+}\left(\bar{p}_{w}, \bar{\lambda}\right) \ell_{i}^{-}\left(p_{\ell}, \alpha_{\ell}\right) \ell_{j}^{-}\left(\bar{p}_{\ell}, \bar{\alpha}_{\ell}\right)$. Attaching the legs of the four final particles to the two $N$-propagators differently leads to the other contributing (tu-channel) diagrams. The CMS squared energy of the process is $s=(p+\bar{p})^{2}$. 


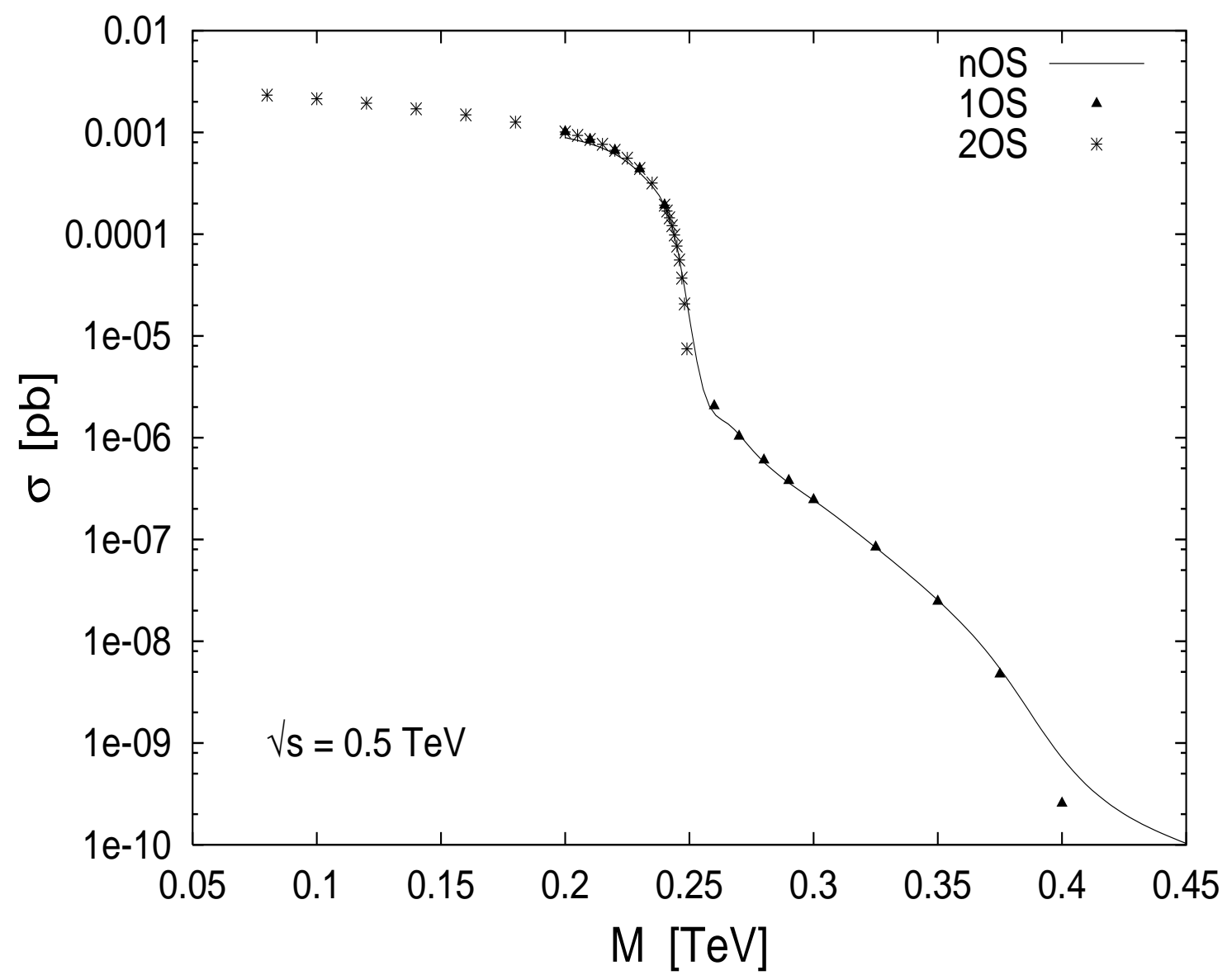

FIG. 2. Sum of the total cross sections for the reactions $e^{-} \mu^{+} \rightarrow N N \rightarrow W^{+} W^{+} \ell_{i}^{-} \ell_{j}^{-}(i, j=1,2,3$; $\ell_{1}=e, \ell_{2}=\mu, \ell_{3}=\tau$ ), as function of the Majorana neutrino mass $M$, for the CMS process energy $\sqrt{s}=0.5$ $\mathrm{TeV}$. Summation over the final, and average over the initial polarizations were carried out. The values of the "mixing" parameters $H^{\prime}$ and $H$ of Eq. (2) were set equal to the upper bounds (7). The results of the general (nOS) program are displayed as full lines, in various kinematic regions. The results of the simpler 1OS program are displayed as triangles, in the $1 \mathrm{OS}$ and partly in the $2 \mathrm{OS}$ kinematic regions. The results of the short 2OS program are displayed as crosses, in the $2 \mathrm{OS}$ kinematic region. 


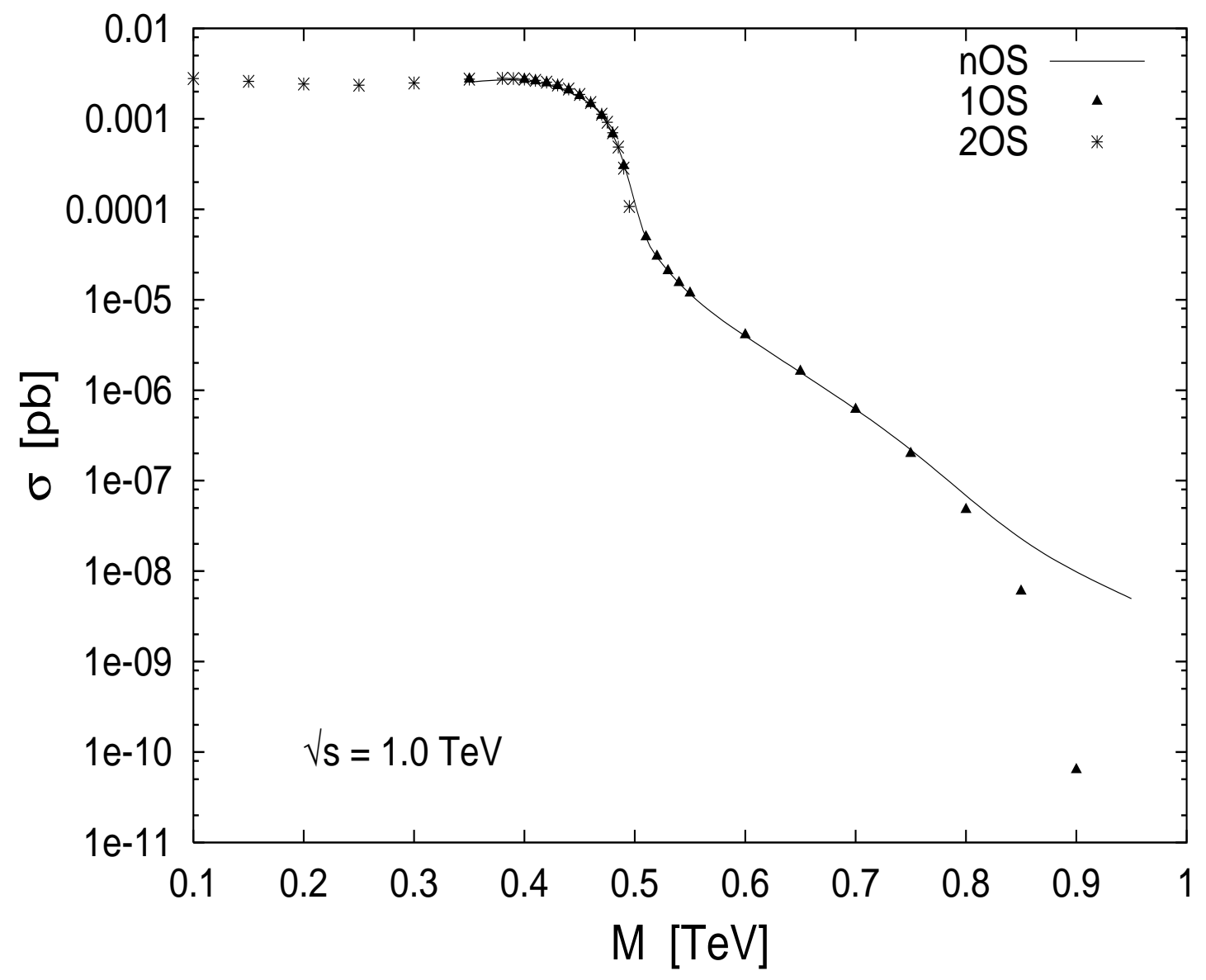

FIG. 3. Same as Fig. 2, but for $\sqrt{s}=1.0 \mathrm{TeV}$. 


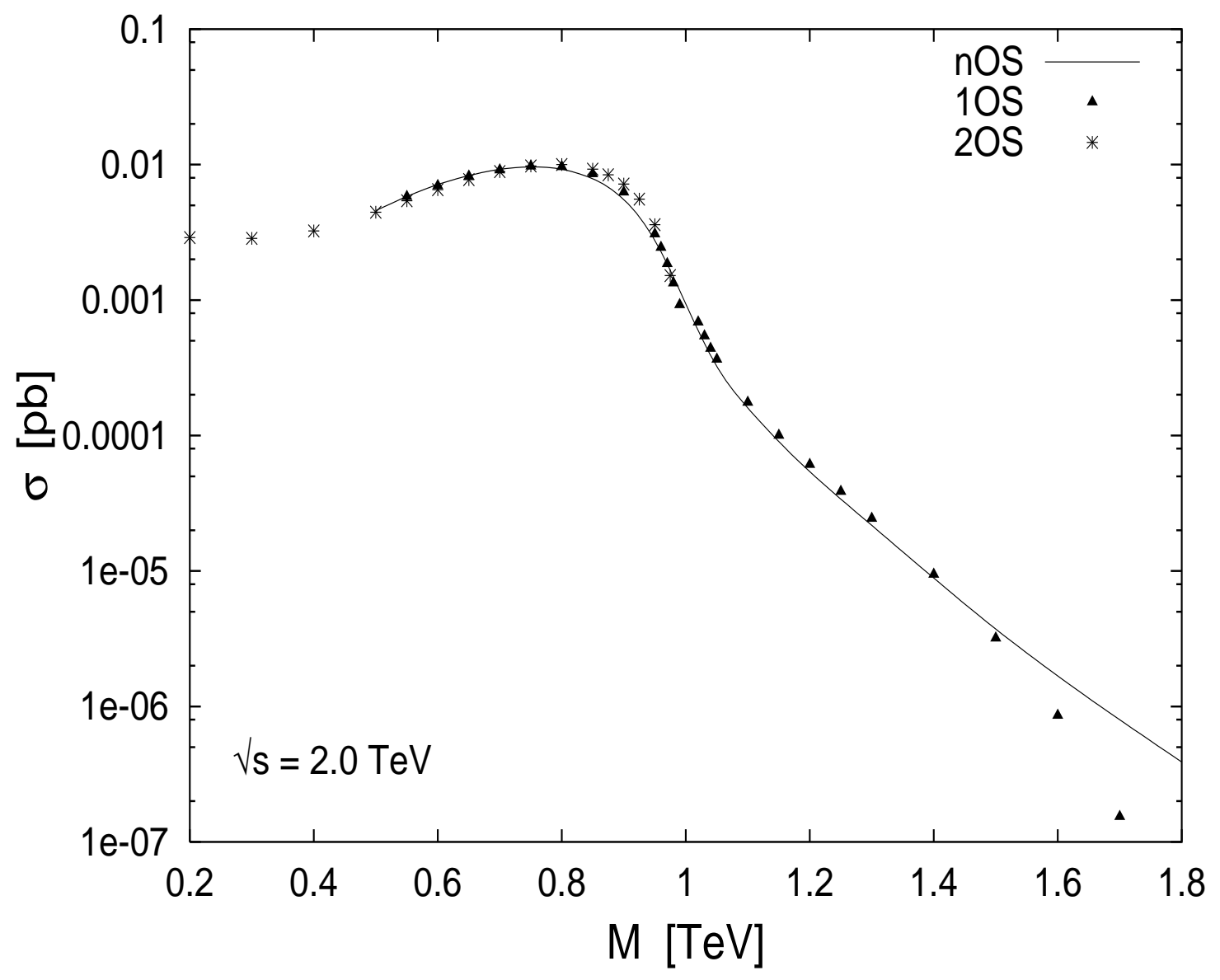

FIG. 4. Same as Fig. 2, but for $\sqrt{s}=2.0 \mathrm{TeV}$. 


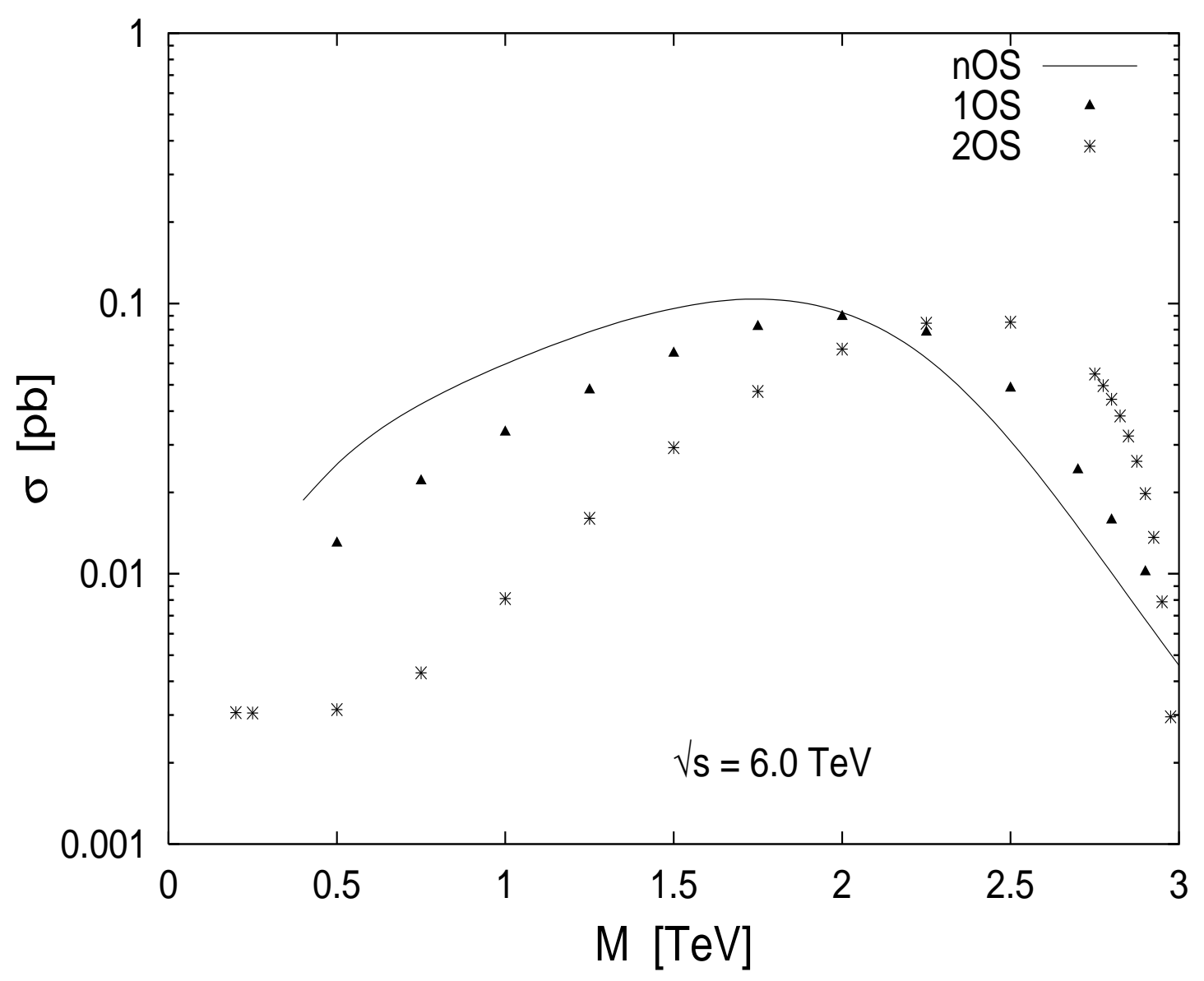

FIG. 5. Same as Fig. 2, but for $\sqrt{s}=6.0 \mathrm{TeV}$. 


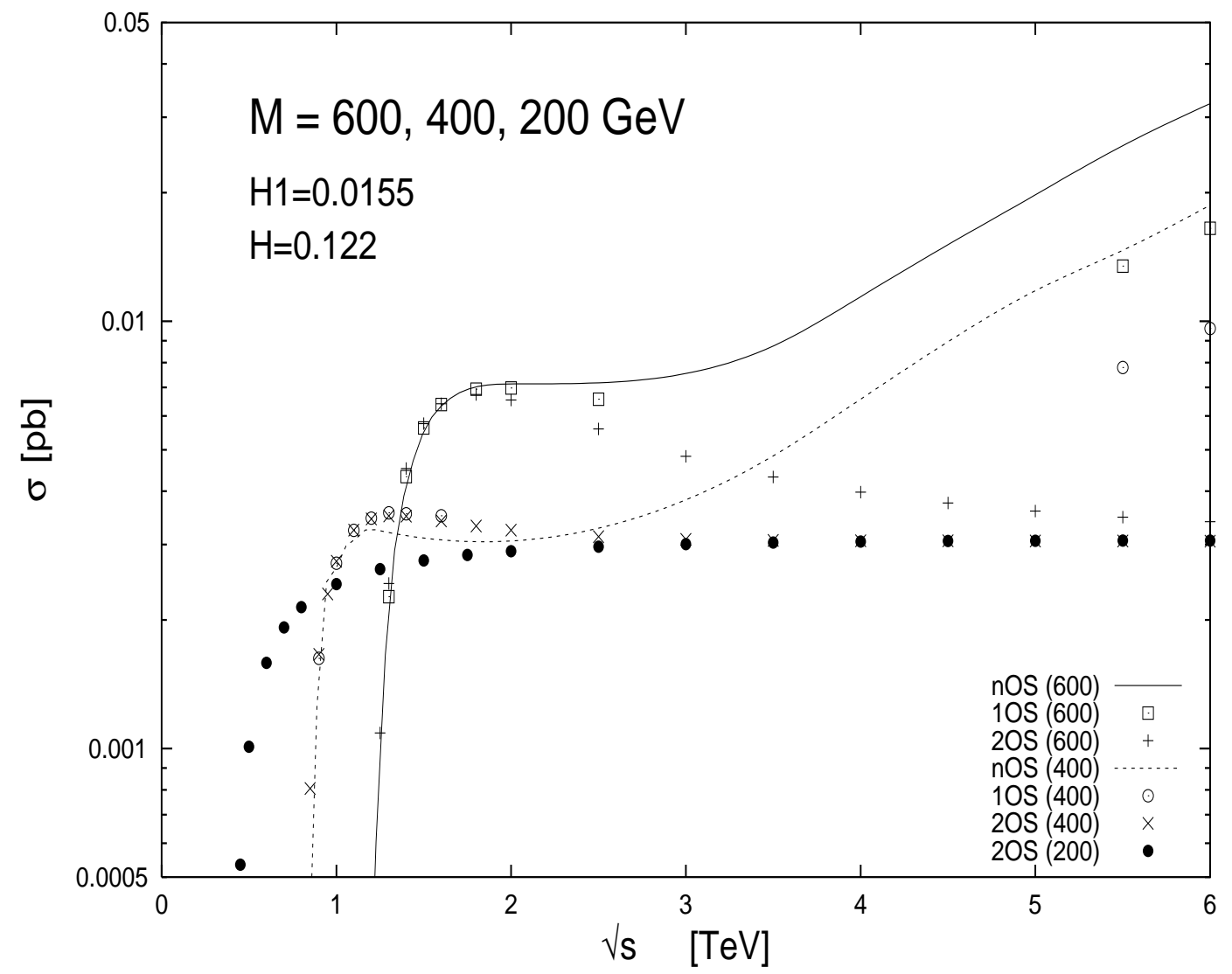

FIG. 6. Sum of the mentioned total cross sections for the reactions $e^{-} \mu^{+} \rightarrow N N \rightarrow W^{+} W^{+} \ell_{i}^{-} \ell_{j}^{-}$ $\left(i, j=1,2,3 ; \ell_{1}=e, \ell_{2}=\mu, \ell_{3}=\tau\right)$, as function of the CMS process energy $\sqrt{s}$, for Majorana neutrino mass $M=600,400$ and $200 \mathrm{GeV}$. 